\title{
Gathering the Users' Needs in the Development of Assistive Technology: A Blind Navigation System Use Case
}

\author{
Hugo Paredes, Hugo Fernandes, Paulo Martins, and João Barroso \\ INESC TEC (formerly INESC Porto) and University of Trás-os-Montes e Alto Douro, \\ Quinta de Prados, Apartado 1013, Vila Real, Portugal \\ \{hparedes, hugof, pmartins, jbarroso\}@utad.pt
}

\begin{abstract}
Assistive technology enables people to achieve independence in the accomplishment of their daily tasks and enhance their quality of life. However, the development os assistive technology does not always follow user needs and expectations, comprising their usability and effectiveness. This paper discusses the design and evaluation strategies for assistive technologies applied to a blind navigation system case study. The research carried out focused on the gathering of user requirements for ensuring enhanced autonomy of blind people in their daily life. The picked requirements were used in an architecture that unifies the benefits of a redundant blind navigation system with a set of services that are provided by daily used information and communication technologies. The system combines guidance, navigation and information gathering, extending traditional aids with realtime knowledge of the surrounding environment to provide an enhanced assistive tool for autonomy of the blinds. Moreover, the developed solution fills the gap of existing solutions that require the users to carry a wide range of devices and, mostly, do not include mechanisms to ensure the autonomy of users in the event of system failure.
\end{abstract}

Keywords: usability evaluation, interviews, blindness, assistive technology, navigation, autonomy.

\section{Introduction}

"Assistive Technology is any object or system that increases or maintains the capabilities of people with disabilities" [7]. However, little proof exists to support the real benefits of assistive technology both for the researchers and the users, and the contention that users are satisfied with relevant aspects of these assistive technologies 21. Moreover, there are well known problems associated with the design and development of assistive technologies that have been widely discussed by the community. Authors explore the agenda, development and value of assistive technology from different perspectives, from its development to the users' satisfaction [7/21/6].

\footnotetext{
${ }^{1}$ According to the Assistive Technology Act of 1998, S.2432.

C. Stephanidis and M. Antona (Eds.): UAHCI/HCII 2013, Part III, LNCS 8011, pp. 79 88, 2013.

(C) Springer-Verlag Berlin Heidelberg 2013
} 
Following the development of a core blind navigation system in the SmartVision project, the evaluation of the prototype revealed that further requirement analysis was needed [9]. Some of those requirements were introduced in the nav $4 \mathrm{~b}$ prototype that preceded the Blavigator project [10]. However, the technological solutions introduced required a shift in the research strategy and the usage of new methodologies to enhance the gathering of user requirement and their real needs. The major research questions that arise at those stages concern: (1) the context where blind or visual impaired users need to use a navigation system; (2) how the users interact with the system; (3) the availability of the system; and (4) how and why the application helps the users to enhance their autonomy. In order to address these questions a study with visually impaired users was developed, which sought the evaluation of their daily habits in order to examine how assistive technology might be an aid in their autonomy. This approach follows the principles of User-Centred Design [22, where "technology related policies and services need to emphasise consumer involvement and their long-term needs to reduce device abandonment and enhance their satisfaction" [16].

This paper presents the exploratory study of user requirements in the Blavigator project for blind navigation. The methodology used for the study is analyzed and discussed, followed by an explanation of the work developed. The core of the study is a semi-structured interview that was conducted with users with different levels of blindness. The analysis methodology used to gather data from the interviews [23 2 25] allowed reevaluating the system requirements and weaving new directives on the real needs of users for a blind navigation system. The process of analysis and development of accessible applications is discussed taking into account the experience gained from this use case and the analysis of reference bibliography in the domain. The discussion follows with the lessons learned and the needs to be addressed. The presented study reveals that, as in similar studies 4], users' input is an important component to help guide designers in producing assistive technologies. Moreover, the choice of the evaluation methodology follows the HCI research patterns and "must arise from and be appropriate for the actual problem or research question under consideration" 11. A final lesson regards the presence of the users in every phase of the development of assistive technologies, in order to ensure that the system actually behaves as we expect and meets their requirements [6].

The structure of the remaining paper is as follows: Section 2 describes background and related work. In section 3, the Blavigator project is presented highlighting its features and major requirements. The fourth section focuses on the research methods applied in this study. Section 5 gives an overview of the empirical findings gathered from the field work. A discussion of the empirical results is presented in Section 6. The last section provides some final remarks.

\section{Background}

Assistive technologies play an important role in the lives of many people by providing the means to perform their daily living activities and helping people 
with disabilities to achieve greater independence and enhance their quality of life. From the various assistive technologies available nowadays, a special focus was put on those related with mobility. Mobility is a fundamental part of life for every human and heavily depends on sight for orientation and navigation. However, blind and visually impaired people need to rely on other senses, such as touch and hearing, to ensure their mobility [17.

Current technology enabled the creation of systems that assist people with special needs to navigate, eliminating many of their mobility restrictions [14 24]. The development of such systems has been discussed by several authors [26]. Most of these studies evaluate the needs and requirements of electronic travel aids. Sanchez '[19] argues that this systems should not focus on the replacement of traditional assistive tools, as the white cane, since it is "very unlikely to redesign such a device that is cheap, portable and useful". According to the author, efforts should be made in order to detect and inform users about obstacles and provide more accurate information about the environment. In a user centric perspective, Pressl and Wieser [18] worked in a project with the collaboration of blind people in order to develop "a prototype of a navigation system which covers the overall spectrum of navigational components". Polacek et al. 17] follow the same perspective, conducting an in-depth evaluation with blind users in order to evaluate the relevant aspects of navigation for the blind. In their study, the authors also focus the integration of "sensors for position and rotation acquisition, algorithm for route computation and an interface allowing the user to control the navigation system".

In the last decade several projects have emerged aiming to enhance indoor navigation [8]. The BIGS System, proposed $\mathrm{Na}$ [15], includes an infrastructure, the smart floor, and a portable terminal unit. A more complex system was presented by Ding 5] consisting of RFID tags, a portable reader which can be integrated into the white cane, a mobile phone, a Call Center and a central information server. Jacquet et al. 13 developed a system based on a white cane with a laser telemeter and GPS, that provides guidance information as well as descriptive details about places based on semantic descriptions. Recently, Chen 3] proposed the inclusion of pre-built RFID tags in blind pathways. Moreover, the SmartVision project aims to develop a system for assisting the blind navigate autonomously, integrating GPS, Wi-Fi, RFID and computer vision technology [1. In a complementary perspective, the TANIA system, which was originally developed to provide the blind with a navigation device, was extended with an RFID reader or the recognition of tagged objects [20]. The integration of navigation and object recognition was previously explored by Hub et. al[12.

\section{The Blavigator Project}

The navigation for the blind has been shown as an area of interest in the scientific community across the last few decades. Some of the issues associated with these systems are generic assistive technologies, and other related to the specific context of the case study. For the last years, our team has developed prototypes 
of navigation systems for the blind in order to solve some of the problems associated with these systems. On the genesis of the current Blavigator project, which integrates the study presented herein, is the Smartvision project.

The main goal of the SmartVision project was to develop and integrate technology for aiding blind users and those with severe visual impairments into a small portable device that is cheap and easy to assemble using off-the-shelf components. This device should be extremely easy to carry and use, yet providing all necessary help for autonomous navigation. It should be stressed out that the device was designed to be an extension of the white cane, not a replacement, and to be "non-invasive", issuing warning signals when approaching a possible obstacle, a point-of-interest or when the footpath in front is curved and the heading direction should be adapted. In this sense, the SmartVision prototype addressed three main applications: (1) local navigation for centring on footpaths etc. and obstacle avoidance, in the immediate surroundings, but just beyond the reach of the white cane; (2) global navigation for finding one's way; and (3) object/obstacle recognition, not only on the shelves in a pantry or supermarket, but also outdoor: bus stops, taxi stands, cash machines (ATM) and telephone booths.

Based on one of the technologies of the infrastructure of the SmartVIsion, the Radio Frequency IDentification (RFID), used to create electronic trails and tags, the Nav4B system [10], proposed the integration of blind navigation with day-to-day life supporting information systems, as traffic lights and public transportations. Moreover, the system focus on the ergonomics and ubiquity go the solution, using typical blind aids: a white cane; a smartphone; and bone- conduction headphones.

The Blavigator project extends the main goals of the SmartVision project with the concept of the Nav4B architecture. In other words, the Blavigator prototype intends to be an integrated navigation system which increases the autonomy of daily life significantly while being, at the same time, cheap, reliable, small and easy to use. Therefore the hardware platform chosen for the Blavigator prototype was a smartphone. This platform embeds all the sensors required, like stereo vision, digital compass and a GPS antenna, and also provides all the data connections needed, like Internet connection and Bluetooth for interfacing with the instrumented white cane. Moreover, in the development of the Blavigator project an extensive study of user requirements was performed as detailed described in the next sections.

\section{Research Methods}

The experience acquired in the development of navigation systems for the blind demonstrated the importance of assessing user needs. However, as in the bulk of computer systems, users do not know their needs accurately, the study and requirements gathering goes through the analysis of their habits and how the technology shall be embedded in their everyday life. Research methodologies for conducting such studies are diverse, using, among others, ethnographic techniques, interviews and surveys. 
In this sense, the research method used was a semi-structured interview that provides a closer interaction with the existing sample. This methodology ensures greater richness and quality of information collected, to allow direct contact and interaction with the sample during the interview, contrasting with other methodologies based on surveys. Moreover, the use of ethnographic techniques required special conditions which could hardly be guaranteed (such as the ethnographer be blind so be able to experience the same experiments and thus report).

A semi-structured interview is characterised by the existence of a previously prepared script that serves shaft guiding the development of the interview, ensuring that the various participants answer the same questions without following a strict order to adapt the interview development to the interviewee.

The selection of the users for the sample group should ensure that they represent a subset of the study universe, to guarantee the scope of the study, the search universe and the data sources in conjunction with the time and funds available. Thus, a sample set of five individuals male and female, from different age ranges and visual impaired or legally blind were selected.

In order to characterise the sample group in detail, the interview guide began by gathering the age, sex, origin of visual impairment or legally blind interviewee. The script also contained indications to the interviewer to ask for the permission of the interviewee to perform the audio recording of the interview for later analysis of more reliable data collection ensuring his/her privacy rights. Following the set of objectives for the study, the interview guidelines included questions about the use of devices and assistive technologies for enhancing the mobility and independence of blind users, as other everyday use objects. To measure the degree of autonomy of the individual, the interview also focused on urban obstacles and barriers, whether fixed or mobile. Additionally, and when possible, individuals were questioned in relation to the degree of nuisance caused by those barriers to their daily lives and the environments in which they arose (known / unknown, interior / exterior). Therefore it was ensured that a perspective of the wider difficulties experienced by each individual was gathered. For a better characterisation of the individuals' knowledge about the assistive technologies for describing the environment, stakeholders were asked about their experience in usage of tactile flooring, traffic lights with sound and subtitles in braille (for example at bus stops). This nuclear base of information helps to understand the level of independence, autonomy and knowledge of each individual in this domain. Finally, the script contained questions guidelines that aim to find the ideal solution to provide autonomy to users. However, the questions should not be directly asked to the user, but presented with various types of answers and without limitation. That strategy allows the individual the opportunity to express themselves by using situations they experienced.

\section{$5 \quad$ Empirical Findings}

The interviews were conducted in an informal and familiar environment to the interviewee. This methodology promotes the interview to arise as an informal 
conversation, having some points on which the interviewer should conduct the interview, but always ensuring freedom of the interviewee to express their opinions. Consequently, and depending on the individual interviewed, the duration of the interviews ranged between 105 and 15 minutes.

The characterisation of the interviewed individuals revealed that two intervenients were legally blind and the remaining three were visual impaired. The legally blind individuals lost their vision during their life, one at 24 and the other at 20 years of age, and are currently 32 and 38 years old respectively. As the blind individuals were not born blind, they had to adapt to a new reality. In the general context, they seem well adapted to this reality and are very independent individuals without any shame to ask and answer any question about any subject. Moreover they pursue an active life, personal and professionally, and are very curious about new technologies which can increase their autonomy, saying that "I really like being autonomous and I am volunteer for new experiences." Meanwhile, visual impaired individuals have been losing their vision gradually throughout their life. All individuals consider living in quiet towns. Some of them often use public urban transportation. One of the individuals reported that she only uses public transport when travelling to large cities, because she needs to.

The usage of mobile phone is transversal to all the surveyed users. The choice of all of them fell on the same brand as it was one of the first to provide a screen reader for these devices. Among the various functions available the interviewees make calls, store phone numbers and events on the agenda. In everyday life all of the interviewed blind individuals use the white cane, although one of them was waiting for a seeing eye dog to ensure increased mobility and autonomy. The usage of white cane was not as common among the partially sighted surveyed, thus their mobility was more conditioned. The clock is also an object of everyday use, in its various versions: audio, tactile, etc..

The opinion of respondents is unanimous concerning the amount of existing barriers, as there are numerous urban barriers, particularly poles, dustbins, letterbox, public telephones, terraces and products displayed on the sidewalk in traditional stores. The barriers are not only found in external areas, but also inside buildings, where often information is scarce, particularly regarding the location of services and identification of stairs and elevators. Despite the barriers faced, individuals are also unanimous in considering that in already known paths of their daily life, obstacles and their liable locations are easily detected, even if they are just temporary obstacles.

Regarding the existing aid individuals are skeptical and rely more on their senses than on the information provided by electronic devices. For example, in the case of traffic lights with audio information one of the users stated: "who can assure me that the traffic lights are working well and when I cross the street I will not be hit by a vehicle?" However there are situations in which Assistive technology may be useful as one of the users remarked by saying: "I like the automatic doors more because the recognise us when we arrive". The nonexistence of information is another problem mentioned: "public transports lack indications about the destinations, I often have to ask the driver". 
As for navigation in familiar surroundings blind individuals report that since reference points are not changed, they feel completely free to walk without a cane. The situation turns out to be not much different on the outside, showing that they rediscovered the cities where they live just with the help of their white cane. One adds: "I travel alone on buses, usually recognize the path by the movement of the bus and easily know my location". Still on his autonomy the other individual states: "I can go anywhere, I quite manage my autonomy and mobility". The independence / autonomy of partially sighted individuals is different, having been noted that, for example, one of the individuals has a higher dependence mainly from her mother who carries and accompanies her everywhere. A revealing fact was to find that in unknown areas, outside her hometown, she does not have any problem in using her white cane. However in her hometown she is a bit more retracted "when I go into another town, like Oporto, I use a cane but here I do not use it. I am ashamed."

\section{Discussion}

In general, all individuals surveyed are accompanied by the same objects, just like any other person without any special need. The objects are the personal mobile phone, laptop computer, watch, keys, and obviously in the case of the blind, the white cane. Therefore, mobility and autonomy assistive technologies must be integrated in objects that are common in the everyday life of their users.

User requirements also include information about architectural barriers and movable obstacles that should be communicated to users so that they can choose alternative paths / routes. The lack of information about the surrounding environment is a key problem described by users, also requiring information on specific locations, particularly inside the buildings. The needs are extensible to services, one of the key problems identified being the lack of information in the public transports.

It is also worth mentioning that the study carried out is not conclusive about how the indications and information can be delivered to the users. None of the respondents were explicit in their preferences, and the results were conditioned by the hypotheses they were given during the interview. The interviewees are nonetheless broadly in agreement on this point: the indications should be clear and concise and more ancillary information should be available optionally, although it would relay more security to the individual if the path was done along a wall in order to always have feedback for orientation, instead of traversing the space without reference points.

Finally, one of the most prominent aspects of the study relates to the need for usage of a system to support mobility and navigation for the blind. In everyday life users use the same pathways, the same schedules, knowing the environment that surrounds them very well, so there is no need for any information other than that relating to urban temporary barriers and obstacles that may arise. Consequently, the usage of a navigation system for the blind will be equal to their usage for a normal person, that is, when moving to an unknown location. 
Some of the problems highlighted in this study are common to previous studies, in which we studied the habits and daily life of users [1917. Despite the variety and richness of the information gathered, the need to perform functional tests with prototypes is clear, in order to evaluate the adaptation of the system used and its usage.

\section{Final Remarks}

The improvement of the functional capabilities of people with special needs is often achieved through the use of assistive technologies. These technologies ensure an improvement of the general welfare of the people, enhancing their capabilities. However, their design is sometimes limited. Some of the solutions are too specific and therefore have very high costs. Other solutions are designed to ensure universal access, with features that allow its usage by a broad population. Nevertheless, and particularly in this last case, some of the solutions are designed in the laboratory without examining the real needs of individuals.

In the work presented in this paper we highlight the need for interaction with users to gather the system requirements for assistive technologies. In order to illustrate this need a navigation system for the blind is presented as a case study. The system has evolved in order to narrow down its requirements according to the users needs.

In the last iteration of the system development semi-structured interviews were conducted with potential users in order to understand their daily habits and thereby assess the requirements of a system that supports them in their mobility. The research methodology used proved suitable to the study, particularly by creating an informal atmosphere with the interviewees and not target their responses to the objective of the ongoing work. Therefore the problems and the real needs of users in their day to day life were perceived and the requirements for the enhancement of the system were gauged, in order to increase their autonomy and mobility.

The work carried out showed some limitations, namely at the level of obtaining information from users concerning what they would expect in a system to support their mobility.

Furthermore, the need for the evaluation of the development process as a whole, ie, a complete development cycle, from the requirements elicitation until the prototype testing, is also evident,. Therefore the main purpose of future work is to understand if the whole process of development was actually adjusted to the user needs, performing applicational tests with the same sample group using a prototype navigation system that reflects the needs evidenced in the study.

Acknowledgements. The Portuguese Foundation for Science and Technology (FCT) supported this research through the project RIPD/ADA/109690/2009 - BLAVIGATOR: a cheap and reliable navigation aid for the blind. We would also like to thanks the undergraduate and graduate students of the University of Trás-os-Montes e Alto Douro, which were involved in this process, for their support. 


\section{References}

1. du Buf, J.H., Barroso, J., Rodrigues, J.M., Paredes, H., Farrajota, M., Fernandes, H., ao José, J., Teixeira, V., Saleiro, M.: The smartvision navigation prototype for blind users. JDCTA 5(5), 351-361 (2011)

2. Burnard, P.: A method of analysing interview transcripts in qualitative research. Nurse Education Today 11(6), 461-466 (1991)

3. Chen, J., Li, Z., Dong, M., Wang, X.: Blind path identification system design base on rfid. In: 2010 International Conference on Electrical and Control Engineering (ICECE), pp. 548-551 (June 2010)

4. Choi, Y.M., Sprigle, S.H.: Approaches for Evaluating the Usability of Assistive Technology Product Prototypes. Assistive Technology 23, 36-41 (2011)

5. Ding, B., Yuan, H., Zang, X., Jiang, L.: The research on blind navigation system based on rfid. In: International Conference on Wireless Communications, Networking and Mobile Computing, WiCom 2007, pp. 2058-2061 (2007)

6. Dix, A., Finlay, J.E., Abowd, G.D., Beale, R.: Human-Computer Interaction, 3rd edn. Prentice-Hall, Inc., Upper Saddle River (2003)

7. Edyburn, D.: Rethinking assistive technology. Special Education Technology Practice 5(4), 16-23 (2004)

8. Faria, J., Lopes, S., Fernandes, H., Martins, P., Barroso, J.: Electronic white cane for blind people navigation assistance. In: World Automation Congress (WAC), pp. 1-7 (September 2010)

9. Fernandes, H., Conceição, N., Paredes, H., Pereira, A., Araújo, P., Barroso, J.: Providing accessibility to blind people using gis. Universal Access in the Information Society 11(4), 399-407 (2012)

10. Fernandes, H., Faria, J., Paredes, H., Barroso, J.A.: An integrated system for blind day-to-day life autonomy. In: The Proceedings of the 13th International ACM SIGACCESS Conference on Computers and Accessibility, ASSETS 2011, pp. 225226. ACM, New York (2011)

11. Greenberg, S., Buxton, B.: Usability evaluation considered harmful (some of the time). In: Proceedings of the SIGCHI Conference on Human Factors in Computing Systems, CHI 2008, pp. 111-120. ACM, New York (2008)

12. Hub, A., Diepstraten, J., Ertl, T.: Design and development of an indoor navigation and object identification system for the blind. In: Proceedings of the 6th International ACM SIGACCESS Conference on Computers and Accessibility, ASSETS 2004, pp. 147-152. ACM, New York (2004)

13. Jacquet, C., Bellik, Y., Bourda, Y.: Electronic locomotion aids for the blind: Towards more assistive systems. In: Ichalkaranje, N., Ichalkaranje, A., Jain, L.C. (eds.) Intelligent Paradigms for Assistive and Preventive Healthcare. SCI, vol. 19, pp. 133-163. Springer, Heidelberg (2006)

14. Kitsas, I.K., Panoulas, K.J., Kosmidou, V.E., Taplidou, S.A., Saragiotis, C.D., Hadjileontiadis, L.J., Panas, S.M.: Smarteyes: An efficient mobile phone/navigator for blind or visually impaired people. In: Proceedings of the Forum for the ICT Professionals Congress, FITCE 2006 (2006)

15. Na, J.: The blind interactive guide system using rfid-based indoor positioning system. In: Miesenberger, K., Klaus, J., Zagler, W.L., Karshmer, A.I. (eds.) ICCHP 2006. LNCS, vol. 4061, pp. 1298-1305. Springer, Heidelberg (2006)

16. Phillips, B., Zhao, H.: Predictors of assistive technology abandonment. Assistive Technology: the Official Journal of Resna 5(1), 36-45 (1993) 
17. Poláček, O., Grill, T., Tscheligi, M.: Towards a navigation system for blind people: a wizard of oz study. SIGACCESS Access. Comput. 104, 12-29 (2012)

18. Pressl, B., Wieser, M.: A computer-based navigation system tailored to the needs of blind people. In: Miesenberger, K., Klaus, J., Zagler, W.L., Karshmer, A.I. (eds.) ICCHP 2006. LNCS, vol. 4061, pp. 1280-1286. Springer, Heidelberg (2006)

19. Sánchez, J., Elías, M.: Guidelines for designing mobility and orientation software for blind children. In: Baranauskas, C., Abascal, J., Barbosa, S.D.J. (eds.) INTERACT 2007. LNCS, vol. 4662, pp. 375-388. Springer, Heidelberg (2007)

20. Schmitz, B., Hub, A.: Combination of the Navigation System TANIA with RFIDBased Initialization and Object Recognition, p. 2 (2009), http://www.icevi-europe.org/dublin2009/index.html

21. Stickel, M.S., Ryan, S., Rigby, P.J., Jutai, J.W.: Toward a comprehensive evaluation of the impact of electronic aids to daily living: Evaluation of consumer satisfaction. Disability and Rehabilitation: An International, Multidisciplinary Journal 24(1-3), $115-125$ (2002)

22. Vredenburg, K., Mao, J.Y., Smith, P.W., Carey, T.: A survey of user-centered design practice. In: Proceedings of the SIGCHI Conference on Human Factors in Computing Systems, CHI 2002, pp. 471-478. ACM, New York (2002)

23. Wengraf, T.: Qualitative research interviewing: biographic narrative and semistructured methods. Sage, London (2001)

24. Willis, S., Helal, S.: Rfid information grid for blind navigation and wayfinding. In: Proceedings of the Ninth IEEE International Symposium on Wearable Computers, ISWC 2005. IEEE Computer Society, Washington, DC (2005)

25. Witzel, A.: The problem-centered interview. Forum Qualitative Sozialforschung / Forum: Qualitative Social Research 1(1) (2000)

26. Zhang, J., Ong, S.K., Nee, A.Y.C.: Navigation systems for individuals with visual impairment: a survey. In: Proceedings of the 2nd International Convention on Rehabilitation Engineering \& Assistive Technology, iCREATe 2008, Singapore Therapeutic, Assistive \& Rehabilitative Technologies (START) Centre, Kaki Bukit TechPark II, Singapore, pp. 159-162 (2008) 